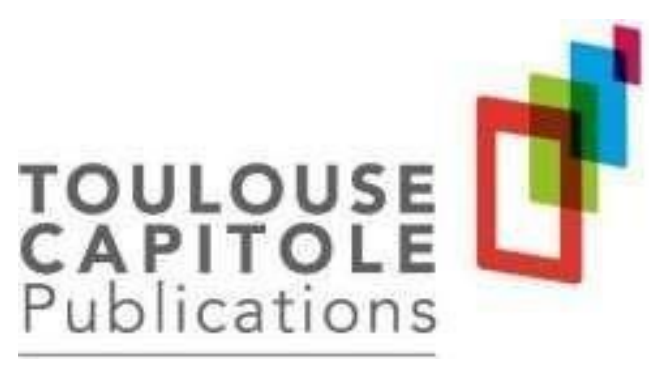

« Toulouse Capitole Publications » est l'archive institutionnelle de l'Université Toulouse 1 Capitole.

Chronique de fiscalité immobilière 2020

\author{
RAIMBAUlt DE Fontaine SOPHIE
}

Référence de publication : RaImBault DE Fontaine (S.), «Chronique de fiscalité immobilière 2020 », Droit et ville, vol.2 (n90), 2020, p. 169-187. 


\section{Chronique de fiscalité immobilière 2020}

Pour des raisons évidentes de format, les développements qui suivent, et qui ne prétendent nullement à l'exhaustivité, se concentreront sur les problématiques qui ont paru les plus intéressantes. Bien que des nouveautés soient également intervenues en matière de revenus fonciers, de plus-values immobilières ou même d'IFI, le choix a été fait de consacrer cette première livraison de notre chronique annuelle de fiscalité immobilière à l'actualité jurisprudentielle qui, en 2020, a été particulièrement riche en matière de TVA et de droits d'enregistrement.

\section{TVA ET SECTEUR DE L'IMMOBILIER}

A. LOCATION D'IMMEUBLES NUS À USAGE PROFESSIONNEL, PORTÉE DE L'OPTION PoUR LA

TVA

Aux termes du $2^{\circ}$ de $1^{\prime}$ article $261 \mathrm{D}$ du CGI, les locations de terrains non aménagés et de locaux nus, sont exonérées de TVA. Néanmoins, en application du $2^{\circ}$ de l'article 260 du même code, « les personnes qui donnent en location des locaux nus pour les besoins de l'activité d'un preneur assujetti à la taxe sur la valeur ajoutée ou, si le bail est conclu à compter du 1er janvier 1991, pour les besoins de l'activité d'un preneur non assujetti » peuvent sur leur demande acquitter la taxe sur la valeur ajoutée. La location de locaux nus destinés à l'habitation ou à un usage agricole, ou à un preneur non assujetti, sauf lorsque le bail fait mention de l'option du bailleur, exclut toute option pour la TVA. L'article 193 de l'annexe 2 du CGI prévoit quant à lui que « dans les immeubles ou ensembles d'immeubles comprenant à la fois des locaux nus donnés en location ouvrant droit à l'option en application du $2^{\circ}$ de l'article $260 \mathrm{du}$ CGI et d'autres locaux, l'option ne s'étend pas à ces derniers mais elle s'applique globalement à l'ensemble des locaux de la première catégorie ». Sur ce fondement, et par une lecture littérale de l'article précité, la doctrine administrative précise que l'option s'exerce en principe immeuble par immeuble, et que, lorsqu'elle est exercée, " elle couvre obligatoirement tous les locaux non exclus de son champ d'application qu'un bailleur possède dans un immeuble donné » 1 .

Invalidant cette position, le Conseil d'État a jugé par un arrêt SCI EMO du 9 septembre 20202, que les dispositions du $2^{\circ}$ de $1^{\prime}$ article 260 du CGI per- mettent à un contribuable « d'opter pour la soumission à la taxe sur la valeur ajoutée de la location de certains seulement des locaux qu'il exploite dans un même bâtiment. Si elles lui permettent également d'opter pour l'imposition de l'ensemble des locations qu'il réalise dans ce bâtiment, et si dans ce cas, seules celles de ces locations qui portent sur des locaux n'ouvrant pas droit à option restent, le cas échéant, exonérées de taxe sur la valeur ajoutée, elles ne lui en font pas obligation. L'option exercée en vue de la soumission à la taxe sur la valeur ajoutée de la location de certains seulement des locaux d'un même bâtiment n'a pas pour effet de soumettre à cette taxe la location des autres locaux ».

Pour juger que les effets de l'option doivent se limiter aux seuls locaux expressément visés par la lettre d'option - dans l'affaire en cause l'option était accompagnée d'une promesse de bail visant un local particulier - le Conseil d'État s'appuie sur les dispositions de l'article 137 de la directive 2006/112 qui offrent aux États membres la possibilité d'ouvrir au bailleur de biens immeubles une option pour la TVA, alors qu'en application du 1 du I de l'article 135 de ladite directive, les locations d'immeubles nus sont en

principe exonérées. Ce texte, selon les juges du Palais-Royal, ne confère «pas aux États membres la faculté de subordonner à des conditions ou de restreindre de quelque manière que ce soit les exonérations prévues par le 1 de l'article 135, mais leur réserve simplement la faculté d'ouvrir, dans une mesure plus ou moins large, aux bénéficiaires de ces exonérations, la possibilité d'opter eux-mêmes pour la taxation, s'ils estiment 
que tel est leur intérêt ».

Compte tenu de ce qui précède, et pour assurer une lecture de l'article 193 de l'annexe 2 du CGI conforme aux exigences de la directive TVA, le Conseil d'État a considéré que l'application de la TVA à la location d'un local nu ne peut résulter que d'une option expresse du contribuable, ce que confirme d'ailleurs la rédaction du $2^{\circ}$ de l'article 260 du CGI selon lequel l'option se fait sur « une demande » de la personne concernée. D’ailleurs, au-delà des contraintes liées à la primauté de la directive TVA, le rapporteur public Romain Victor soulignait que la position inverse aurait, sans justification, placé les propriétaires de locaux nus situés dans un seul bâtiment dans une situation différente de celle de bailleurs disposant de locaux situés dans des bâtiments distincts. Il ajoutait qu'aucun inconvénient pratique ne s'opposait à cette analyse « bail par bail », les bailleurs optant à la TVA étant d'ores et déjà contraints par les dispositions du $2^{\circ}$ du I de l'article 209 de l'annexe II du CGI de constituer des secteurs distincts d'activité et de comptabiliser, pour l'exercice du droit à déduction, dans des comptes séparés les fractions d'immeubles dont la location est soumise à la TVA et celles pour lesquelles l'option est exclue. On notera que la solution dégagée par le Conseil d'État devrait trouver à s'appliquer à l'option pour la TVA des bailleurs de biens ruraux prévue au $6^{\circ}$ de l'article 260 du CGI, dont l'Administration considère, en l'absence de toute précision textuelle en ce sens, qu'elle revêt nécessairement un caractère global et s'applique à tous les baux consentis par un même bailleur à des agriculteurs3. Néanmoins, la question de sa transposition à cette hypothèse paraît moins aiguë pour les bailleurs en cause puisque leurs locataires sont nécessairement assujettis.

Très libérale, la solution SCI EMO autorise désormais les options individualisées local par local. Elle ouvre aux futurs bailleurs la possibilité d'adapter leur choix à la spécificité de chacun des locaux loués, à celles de leurs locataires tout en tenant compte de leurs droits à déduction, mais aussi le cas échéant, de leur situation au regard d'une potentielle imposition à la taxe sur les salaires et, s'agissant d'immeubles achevés depuis plus de 15 ans, à la contribution sur les revenus locatifs. Elle impose également une rigueur renforcée dans la rédaction des lettres d'option qui devront désigner de manière précise les locaux concernés, notamment lorsque, à l'occasion de leur cession, l'option expresse de l'acquéreur est une condition de l'application du mécanisme de la dispense de l'article 257 bis du CGI4. En effet, lorsque l'acte d'achat mentionne l'engagement de l'acquéreur à opter pour la TVA et comporte une clause couvrant le risque de remise en cause de la dispense par l'Administration, l'acquéreur qui n'opterait pas, ou le ferait de manière incorrecte, engagerait sa responsabilité à l'égard de son vendeur et pourrait être condamné à l'indemniser des conséquences d'un redressement fiscal 5.

La décision SCI EMO pose par ailleurs un certain nombre de questions auxquelles sa seule lecture ne permet pas de répondre 6. S'agissant des baux en cours, il est par exemple permis de s'interroger sur le point de savoir si les bailleurs qui ont été contraints d'opter de manière globale pour la totalité des baux relatifs à des biens situés dans un même immeuble pourraient, pour certains d'entre eux, renoncer à leur option nonobstant le fait que l'article 194 de l'annexe II du CGI prévoit que la dénonciation de l'option ne peut intervenir qu'à partir du premier janvier de la neuvième année civile qui suit celle au cours de laquelle elle a été exercée. En revanche, tant que l'Administration n'a pas révisé sa position exprimée au BOFIP, elle ne pourrait remettre en cause la TVA déduite par le propriétaire bailleur qui, se croyant couvert par une option formulée antérieurement à la décision SCI EMO portant sur l'ensemble d'un immeuble, n'aurait pas explicitement opté lors de l'acquisition d'un nouveau local, le contribuable étant en mesure de lui opposer sa propre doctrine sur le fondement de l'article L. 80 A du LPF. Même si la position de l'Administration doit désormais être lue comme une tolérance administrative autorisant une option globale là où la loi semble imposer des options individualisées, il n'en reste pas moins qu'une mise à jour du BOFIP serait bienvenue.

$B$. LOTISSEUR OCCASIONNEL, NOTION D'ASSUJETTI

Le Conseil d'État s'est, le 9 juin 2020, prononcé sur une affaire dans laquelle une personne, qui avait acquis 
plusieurs parcelles pour les exploiter dans le cadre d'une activité d'arboriculteur, avait, après avoir pris sa retraite, entrepris d'importants travaux de viabilisation et les avaient revendues en dix-huit lots de terrain à bâtir. Le litige portait sur le point de savoir si cette personne physique, qui ne se présentait pas comme exerçant une activité de lotisseur, pouvait être considérée ou non comme un assujetti à la TVA au sens de l'article $256 \mathrm{~A}$ du CGI, de sorte qu'elle aurait dû soumettre les livraisons de terrains à bâtir à la TVA. Confirmant la position de l'Administration, les juges ont indiqué que « la livraison, par une personne physique, de terrains à bâtir est soumise à la taxe sur la valeur ajoutée lorsqu'elle procède, non de la simple gestion d'un patri- moine privé, mais de démarches actives de commercialisation foncière, telles que la réalisation de travaux de viabilisation ou la mise en œuvre de moyens de commercialisation de type professionnel, similaires à celles déployées par un producteur, un commerçant ou un prestataire de services, et qu'elle permet ainsi de regarder cette personne comme ayant exercé une activité économique ». Considérant que les travaux de viabilisation réalisés en l'espèce représentaient plus de $50 \%$ du prix de vente, le Conseil d'État a jugé que « de tels travaux, eu égard à leur importance, ne relevaient pas de la simple gestion d'un patrimoine privé mais caractérisaient l'existence de démarches actives de commercialisation » 7.

Même si l'arrêt ne le mentionne pas expressément, il ressort de sa rédaction et des conclusions du rapporteur public Karin Ciavaldini que le Conseil d'État s'est en réalité approprié les critères dégagés dans la décision Slaby par la Cour de justice de l'Union européenne8 pour déterminer sous quelles conditions un particulier procédant à la cession de terrains à bâtir en dehors de l'exercice de toute activité professionnelle peut être considéré comme un assujetti. Or cette transposition ne s'imposait pas nécessairement. En effet, les critères retenus par la Cour l'ont été aux fins d'identifier les cessions susceptibles de relever du champ d'application de la TVA lorsque l'État membre concerné a décidé d'assujettir à la TVA les cessions de terrains à bâtir réalisées par des assujettis occasionnels, faculté offerte par l'article 12, 1, b de la directive TVA. Or, la France n'ayant pas formulé cette option9, il n'est absolument pas évident qu'une personne physique procédant à la cession occasionnelle de terrains à bâtir puisse, parce qu'elle remplit les conditions posées par l'arrêt Slaby, ipso facto être considérée comme un assujetti au sens de l'article 9, I de la Directive TVA, dont l'article 256 A du CGI assure la transposition en droit français, c'est-à-dire comme exerçant une activité économique, en particulier « l'exploitation d'un bien corporel ou incorporel en vue d'en tirer des recettes ayant un caractère de permanence». Même si la décision Slaby, passablement confuse dans sa rédaction, autorise cette assimilation 10, l'illogisme de la position qui consiste à utiliser des critères similaires pour identifier les assujettis habituels dont les activités relèvent impérativement de la TVA et les assujettis occasionnels dont les opérations ne sont soumises à la TVA que sur option de l'État membre aurait pu justifier que les juges français interrogent la Cour.

Nonobstant cette difficulté, la décision du Conseil d'État valide les indices retenus par la doctrine administrative pour identifier les hypothèses dans les- quelles une personne peut être considérée comme assujettie au titre de la cession isolée d'un bien immobilier. Elle en précise néanmoins les conditions de mise en œuvre d'une manière qui conduit à élargir les hypothèses d'assujettisse- ment de sorte, que tant que l'Administration n'aura pas modifié le BOFIP, certains contribuables pourraient avoir intérêt à se placer sous la garantie de l'article L.80 A du LPF. En effet, si la condition unique pour qu'une telle cession ne relève pas du simple exercice du droit de propriété du cédant correspond, comme dans le BOFIP, à la mise en œuvre " de démarches actives de commercialisation foncière », le Conseil d'État précise que de telles démarches peuvent être caractérisées par la seule réalisation d'importants travaux de viabilisation même si le cédant n'a mis en œuvre aucun «des moyens de vente de type professionnel » similaires à ceux qu'utilisent les opérateurs du secteur de l'immobilier, la vente des terrains ayant, en l'espèce, été confiée à une agence immobilière. Cette position s'écarte donc de la méthode du faisceau d'indices préconisée par l'Administration puisqu'un seul indice, pour peu qu'il soit suffisamment significatif, suffit à justifier l'assujettissement11. Pour les agriculteurs qui cèdent des terrains à bâtir, la démarche du juge remet en cause la position de l'Administration de manière encore plus nette puisque les commentaires publiés au BOFIP précisent que ces cessions peuvent être considérées comme relevant du champ d'application de la 
TVA si, cumulativement, l'exploitant réalise sur ces terrains des travaux significatifs et met en œuvre des moyens de commercialisation avérés (publicité, démarchage) 12. Par ailleurs, et comme l'avait d'ailleurs relevé William Stemmer, l'on peut penser que la rédaction de la décision du Conseil d'État valide l'hypothèse selon laquelle les travaux de viabilisation et les moyens de commercialisation avérés ne sont en réalité que des exemples de démarches actives de commercialisation, de sorte que l'Administration pourrait dans l'avenir exiger que la TVA soit appliquée à des opérations non visées par la doctrine administrative et notamment à des cessions d'immeubles bâtis ayant préalablement à leur cession subi d'impor- tantes transformations 13 .

Enfin, la décision du 9 juin 2020 rappelle utilement que la question de l'assujettissement à la TVA de cessions immobilières réalisées par des personnes ne se présentant pas comme des professionnels de l'immobilier est distincte de celle, pour l'impôt sur le revenu, de la réalisation d'une activité relevant de la catégorie des BIC en application des dispositions du I de l'article 35 du CGI. En effet, dans cette affaire le Conseil d'État a validé les rappels de TVA alors même que la qualification de marchand de biens avait été abandonnée par l'Administration en cours de procédure. Alors que les activités de marchand de biens, de promoteur ou de lotisseur occasionnel, visées respectivement au $1^{\circ}, 1^{\circ}$ bis et $3^{\circ}$ du I de l'article précité, impliquent que les biens cédés aient été dès l'origine acquis pour être revendus, construits ou viabilisés et revendus en lot à bâtir, la condition qui tient à l'intention du contribuable n'est nullement requise en TVA de sorte qu'un particulier pourra parfaitement se trouver contraint de facturer de la TVA alors qu'il sera considéré comme agissant à titre privé pour l'imposition de son revenu.

\section{$C$. TRANSFERT D'UN CONTRAT DE VENTE EN L'ÉTAT FUTUR D'ACHÈVEMENT (VEFA) PORTANT SUR LA LIVRAISON DE LOGEMENTS NEUFS RELEVANT D'UNE OPÉRATION IMMOBILIÈRE Réalisée DANS LE SECTEUR DU LOGEMENT INTERMÉDIAIRE}

Interrogée sur le traitement d'un transfert, cession ou apport en société, d'un contrat de vente en l'état futur d'achèvement portant sur la livraison de logements, l'Administration a, par un rescrit du 28 octobre 202014 apporté des précisions portant d'une part sur la qualification de cette opération en TVA, et d'autre part, sur ses conséquences lorsque le contrat concerne la livraison de logements neufs destinés à la location à usage de résidence principale dans le cadre d'une opération relevant de l'article 279-0 bis A du CGI.

Selon l'article 1601-3 du Code civil « la vente en l'état futur d'achèvement est le contrat par lequel le vendeur transfère immédiatement à l'acquéreur ses droits sur le sol ainsi que la propriété des constructions existantes. Les ouvrages à venir deviennent la propriété de l'acquéreur au fur et à mesure de leur exécution ; l'acquéreur est tenu d'en payer le prix à mesure de l'avancement des travaux ». L'article 1601-14 du Code civil prévoit par ailleurs que « la cession par l'acquéreur des droits qu'il tient d'une vente d'immeuble à construire substitue de plein droit le cessionnaire dans les obligations de l'acquéreur envers le vendeur ».

S'appuyant sur ces dispositions, l'Administration analyse l'opération de cession d'un contrat de VEFA intervenant avant achèvement de l'immeuble comme comportant transfert d'une part de droits immobiliers portant sur un immeuble inachevé, qualifié en TVA de terrain à bâtir s'il est impropre à un quelconque usage15, et d'autre part de la créance d'achèvement de la construction. En conséquence, si la cession est réalisée par un assujetti agissant en tant que tel, elle doit être taxée comme une cession de terrain à bâtir dans la mesure où le $1^{\circ} \mathrm{du}$ I de l'article $257 \mathrm{du}$ CGI prévoit que les droits réels immobiliers suivent le régime du bien immeuble auquel ils se rapportent. La taxe n'est en revanche perçue que sur la valeur du terrain à bâtir, c'est-à-dire sur le prix du terrain et des constructions en cours d'édification. La créance d'achèvement transmise, qui n'est théoriquement pas monétisée, ne donne pas lieu à TVA. Ainsi, le solde du prix encore dû par le cessionnaire au promoteur au titre du contrat transmis et qui sera payé au fur et à mesure de l'avancement des travaux, est en principe exclu de l'assiette de la TVA. Si cette analyse a le mérite de l'orthodoxie civiliste 16, elle étonne néanmoins. Elle diffère de ce que l'Administration semblait avoir 
admis, sans l'avoir expliqué, à propos des cessions de contrats de VEFA lorsque l'avant-contrat avait été signé avant le 31 décembre 2012. Les concernant en effet, il avait été admis de leur appliquer le régime des livraisons d'immeubles acquis comme immeubles à construire tel que prévu par l'article 257 du CGI dans sa version antérieure au 30 décembre 2012, c'est-à-dire que quiconque procédait à leur cession, y compris hors de toute activité économique, était considéré comme assujetti. Ce faisant, la doctrine autorisait le cédant à déduire la TVA acquittée sur les appels de fonds déjà payés mais surtout elle assimilait la cession du contrat de VEFA à la cession de l'immeuble bâti achevé17. Tel n'est plus manifestement le cas.

Interrogée sur le cas spécifique de la cession d'un contrat de VEFA portant sur la livraison de logements neufs destinés à la location à usage de résidence principale dans le cadre d'une opération immobilière réalisée dans le secteur du logement intermédiaire qui, à ce titre, ouvrait droit à la TVA au réduit de $10 \%$ de l'article 279-0 bis A du CGI 18, 1'Administration a également précisé pour répondre aux questions qui lui étaient posées :

- $\quad$ que le taux de $10 \%$ dont a bénéficié le cédant sur les appels de fonds payés au promoteur n'est pas remis en cause et qu'il peut être appliqué aux appels de fonds qui seront réclamés au cessionnaire après transfert du contrat de VEFA, si ce dernier satisfait lui-même aux conditions prévues par l'article 279-0 bis A du CGI, c'est-à-dire notamment s'il s'agit d'un des organismes visés par le dispositif et s'il a obtenu, à titre personnel, l'agré- ment du représentant de l'État 19 exigé ;

- $\quad$ que la TVA ainsi acquittée par le cédant au titre des appels de fonds précédant la cession, qui est considérée comme ayant grevé les éléments du prix d'une opération imposable en aval, à savoir la cession du contrat de VEFA, est déductible dans les conditions de droit commun.

Étrangement pourtant, l'Administration s'est abstenue de préciser le taux de TVA dont relève la cession du contrat de VEFA, alors même que la qualification donnée à cette opération semble exclure que le taux de $10 \%$ prévu par l'article 279-0 bis A du CGI, qui ne s'applique en principe qu'aux « livraisons de logements neufs et logements, issus de la transformation de locaux à usage de bureau », semble ne pas pouvoir concerner une livraison de terrains à bâtir...

$D$.

TVA SUR MARGE

\section{a ) Champ d'application}

Selon l'article 268 du CGI, la TVA peut être liquidée sur la marge, et non sur le prix total, lors de la livraison d'un terrain à bâtir ou d'un immeuble achevé depuis plus de cinq ans lorsque le cédant, assujetti en tant que tel, a opté pour la TVA comme le lui permet le $5^{\circ}$ bis de l'article 260 du CGI. Dans les deux cas, l'acquisition du bien vendu ne doit pas lui avoir ouvert droit à déduction de la TVA20. Les conditions d'application de la TVA sur marge ont, au cours de ces dernières années, donné lieu à un contentieux abondant opposant l'Administration et les professionnels de l'immobilier, promoteurs, lotisseurs et marchands de biens, devant les juges du fond. Le Conseil d'État a, en 2020, rendu un certain nombre de décisions sur les questions juridiques contestées, sans que toutes les incertitudes ne soient pour autant levées.

Précisant les conditions d'application de la TVA sur marge après la réforme de la TVA immobilière en 2010, l'Administration fiscale avait exigé, de manière non anticipée par les professionnels de l'immobilier, deux autres conditions cumulatives à l'application de la TVA sur marge : une condition d'identité physique et une condition d'identité juridique. Dans un certain nombre de réponses ministérielles, elle avait en effet précisé que « la mise en œuvre de ce régime dérogatoire au principe selon lequel la TVA est calculée sur le prix total suppose nécessairement que bien revendu soit identique au bien acquis quant à ses caractéristiques physiques et sa qualification juridique »21. Contestées par les professionnels de l'immobilier, ces deux conditions ont été écartées par les juridictions du fond qui jugeaient qu'il résulte des « dispositions précitées du code général des impôts et de la directive 2006/112/CE du 28 novembre 2006 que l'application de la taxe sur la valeur ajoutée sur la marge aux livraisons de terrains à bâtir est conditionnée au seul fait que 
l'acquisition par le cédant n'a pas ouvert droit à déduction de la taxe sur la valeur ajoutée lors de son acquisition » 22. Prenant partiellement acte de ces condamnations, l'Administration avait, par une réponse ministérielle Vogel du 17 mai 201823 abandonné la condition d'identité physique et admis que « y compris pour les opérations en cours, dans le cas de l'acquisition d'un terrain ou d'un immeuble répondant aux conditions de l'article 268 du CGI qui n'a pas ouvert droit à déduction par un lotisseur ou un aménageur qui procède ensuite à sa division en vue de la revente en plusieurs lots, [que] ces ventes puissent bénéficier du régime de la marge dès lors que seule la condition d'identité juridique est respectée ».

La condition d'identité juridique qui impose que le bien revendu ait une nature juridique identique à celle qu'il avait au moment de son acquisition par le cédant, maintenue par la réponse Vogel, a été réaffirmée dans une réponse ministérielle Falorni de septembre 2019 24, puis validée par le Conseil d'État. Infirmant la position des juges du fond, il en effet jugé, en se fondant sur l'article 392 de la directive 2006/112/CE25 dont l'article 268 du CGI assure la mise en œuvre en droit interne, que les dispositions de ce dernier « s'appliquent aux opérations de cession de terrains à bâtir qui ont été acquis en vue de leur revente et ne s'appliquent donc pas à une cession de terrains à bâtir qui, lors de leur acquisition, avaient le caractère d'un terrain bâti, quand le bâtiment qui y était édifié a fait l'objet d'une démolition de la part de l'acheteurrevendeur »26. Plus clairement encore, il a, au soutien de son refus de saisir le Conseil constitutionnel d'une éventuelle inconstitutionnalité de l'article 268 du CGI, affirmé dans un arrêt du 16 juillet 2020 que ledit article, tel qu'interprété par sa jurisprudence Promialp, implique que «seules les opérations de vente d'un terrain à bâtir acheté comme tel, ou de vente d'un immeuble achevé depuis plus de cinq ans acheté comme tel, peuvent bénéficier des modalités de calcul de la taxe sur la valeur ajoutée qu'il prévoit »27. Le régime de la TVA sur marge est donc réservé aux seules opérations d'achat et revente sans transformation préalable de biens immobiliers : il ne concerne pas la livraison d'un immeuble non neuf que le vendeur aurait construit sur un terrain acquis préalablement. Ce mode particulier de liquidation de la TVA ne trouve pas non plus à s'appliquer lors de la vente d'un terrain à bâtir résultant de la démolition d'un bâtiment, y compris si, lors de son acquisition, l'intention de le détruire avait été clairement formulée 28.

Dans les deux cas précédents, les biens vendus ont objectivement une nature juridique différente de ceux qui avaient été achetés, mais le Conseil d'État vient d'admettre que la condition d'identité juridique impose également que les qualifications juridiques sous lesquelles le bien a été acquis puis revendu soient identiques. Saisi de l'application potentielle de la TVA sur marge aux livraisons de terrains à bâtir issus de la division d'une parcelle sur laquelle figurait au jour de son acquisition un immeuble bâti, il vient de juger que la TVA sur marge ne trouve pas non plus à s'appliquer lorsque « le bien acquis a fait l'objet d'une division parcellaire en vue d'en céder séparément des parties ne constituant pas le terrain d'assiette du bâtiment » 29. Ce faisant, il impose au professionnel revendeur de s'assurer que l'acte par lequel il acquiert un bien en vue d'en revendre la partie libre de toute construction en tant de terrain à bâtir soit établi après une division parcellaire permettant de distinguer la part de l'emprise qui est nue de la part déjà bâtie et de donner à chacune la qualification juridique qui convient30. À défaut de division parcellaire préalable à l'acquisition, le terrain porteur d'un immeuble est en effet, en TVA, qualifié d'immeuble bâti pour la totalité de sa surface 31 et les lots qui en seraient détachés ne pourront être cédés en tant que terrains à bâtir sous le régime de la marge. Nonobstant le fait que les lots vendus n'auraient objectivement pas changé de nature entre leur achat et leur revente, le Conseil d'État confirme donc que ce qui est vendu en tant que terrain à bâtir doit impérativement avoir été acquis sous même qualification juridique pour échapper à la TVA sur prix total.

Cette position très formaliste qui consiste à faire prévaloir la qualification juridique retenue par les parties sur la véritable nature des choses interroge d'autant qu'elle ne résulte pas de manière incontestable du texte de l'article 268 du CGI. Évidemment, elle facilite le contrôle de l'Administration en ce sens qu'elle garantit le traçage des immeubles vendus, et assure que les surfaces cédées en tant que terrains à bâtir présentaient cette caractéristique au moment de leur achat. Lorsqu'elles ont été acquises d'un professionnel, l'identité de qualification assure en outre que les surfaces vendues en terrain à bâtir n'ont pas indûment échappé à la 
TVA lors de leur acquisition. D'ailleurs dans une pareille hypothèse, l'exigence d'identité de la qualification donnée au bien lors des deux opérations successives apparaît même comme un moyen commode de préserver les intérêts du Trésor : alors même que l'Administration ne serait plus dans le délai pour réclamer au premier vendeur la TVA éludée du fait d'une qualification juridique ne correspondant pas à la nature réelle du bien, elle sera perçue à l'étape suivante. Pour autant, une solution plus souple aurait pu être de permettre à l'assujetti de corriger les potentielles erreurs ou imprécisions de qualification commises dans l'acte d'acquisition, à charge pour lui d'établir que la nature des biens en cause n'a pas été modifiée entre leur achat et leur revente. Quoi qu'il en soit, à défaut de pouvoir procéder en amont de l'acquisition à une division parcellaire comme l'exige le Conseil d'État, les professionnels peuvent également, sur le fondement des réponses ministérielles de 2016 précitées32, se contenter d'un document d'arpentage permettant d'identifier les différentes parcelles dans l'acte.

Si les décisions rendues en 2020 par les juges du Palais-Royal ont le mérite de mettre un terme à l'insécurité dans laquelle la position de l'Administration avait placé les professionnels de l'immobilier, il n'est pourtant pas certain qu'elles soldent tout contentieux. Ils viennent en effet de saisir la Cour de justice de l'Union européenne d'un renvoi en interprétation de l'article 392 de la directive TVA, dans le cadre d'un litige dans lequel était en cause l'article 268 du CGI dans sa version antérieure à la réforme de 2010 de la TVA immobilière. La Cour est ainsi sollicitée sur le point de savoir si la TVA sur marge peut être appliquée lorsque l'acquisition du bien vendu n'a pas donné lieu à perception de la TVA, ce qu'admet aujourd'hui la doctrine administrative 33 ou si elle ne peut concerner que les cas où la TVA a bien été perçue mais n'a pas pu être déduite par l'acquéreur. Elle devra aussi confirmer que les livraisons de terrains à bâtir peuvent relever de la TVA sur marge lorsque les terrains en cause n'ont acquis la caractéristique de terrain à bâtir que postérieurement à leur achat par le vendeur, ou lorsqu'ils ont fait l'objet de modifications de leurs caractéristiques telles qu'une division en lots et des travaux de viabilisation34. Autant de réponses qui pourraient évidemment conduire l'Administration et le juge français à modifier sensiblement leurs positions sur l'actuel dispositif de l'article $268 \mathrm{du}$ CGI.

\section{B ) Bases d'imposition et lotisseurs}

Au-delà du champ d'application de la TVA sur marge, ses modalités de calcul ont également donné lieu à des précisions jurisprudentielles récentes lorsque sont en cause des opérations réalisées par des lotisseurs. En principe, selon l'article 268 du CGI, en cas de cession d'un terrain à bâtir, la base d'imposition à la TVA sur marge est constituée par la différence entre le prix exprimé augmenté des charges qui s'y ajoutent et les sommes versées par le cédant pour l'acquisition du terrain ou de l'immeuble. Dans le cadre d'une opération de lotissement, la vente de chaque lot est analysée séparément et, l'administration admet que « le prix d'achat retenu pour le calcul de la base d'imposition propre à chaque lot devra être exactement déterminé à partir du prix d'acquisition des parcelles d'origine en proportion des surfaces incluses dans le lot cédé ». Elle précise par ailleurs que ce prix d'achat comprend la fraction des parties communes éventuellement attachée à chaque lot par tantième, ainsi qu'une part proportionnelle des terrains d'emprise des équipements généraux ou collectifs qui sont remis à la collectivité locale ou à l'association syndicale de propriétaires, souvent pour un prix symbolique 35 .

Cette formulation, pourtant dépourvue de toute ambiguïté, n'a pas empêché le service, dans une affaire dans laquelle un lotisseur avait revendu des terrains à bâtir à des particuliers après les avoir aménagés et viabilisés, de remettre en cause les modalités de calcul de la TVA sur marge au motif que ce dernier aurait, à tort, intégré dans le prix de revient des terrains vendus une quote-part du prix de revient des parcelles non commercialisables destinées aux équipements collectifs du lotissement. La Cour d'appel de Bordeaux a, dans une décision du 9 juillet 202036, jugé, au visa du seul article 268 du CGI, que « bien que non commercialisables, ces surfaces ont représenté pour la société un coût qui doit être pris en compte dans le prix de revient des lots vendus aux particuliers. À cet égard, le prix dont les particuliers, [...], s'acquittent pour l'achat des lots réalisés intègre aussi les tantièmes des parties communes et de l'usage des équipements 
collectifs réalisés par le lotisseur que celui-ci cède gratuitement à la collectivité publique ». Ce faisant, elle a adopté la même position que celle de la Cour d'appel de Lyon formulée dans un arrêt du 25 juin 2019 à propos des terrains d'emprise d'un lotissement qui avait été cédés pour un euro symbolique à la commune pour procéder à l'élargissement de la voie publique37.

\section{E. DÉDUCTION DIFFÉRÉE DE LA TVA PAYÉE LORS DE L'ACQUISITION D'UN IMMEUBLE NON NEUF PLACE EN STOCKS, Y COMPRIS LORSQU'IL EST LOUE EN TVA}

Le Conseil d'État a jugé dans un arrêt Sté Lips et Financière Lord Byron du 27 novembre 2020, sans saisir la Cour de justice de l'Union européenne d'une question préjudicielle comme le demandaient les requérants38, que la TVA supportée par un marchand de biens lors de l'acquisition d'un immeuble non neuf placé en stocks n'est pas déductible immédiatement, quand bien même l'immeuble serait, lors de son acquisition et dans l'attente de sa revente, loué en TVA.

Pour que la TVA qui grève l'acquisition de biens ou de services soit déductible, il convient en principe que celle-ci présente un lien direct avec une opération imposable ouvrant droit à déduction 39. Or s'agissant d'un immeuble acquis alors qu'il était achevé depuis plus de cinq ans, le conseil d'État confirme qu'un tel lien n'existe pas puisque d'une part, le régime d'imposition de sa cession est incertain au jour de son acquisition, et que d'autre part, sa location en TVA ne fait pas naître un lien direct et immédiat avec une opération imposable.

Sur le premier point, la décision confirme pour la première fois que lorsque des immeubles dont la cession est en principe exonérée de TVA sont placés en stocks, c'est-à-dire destinés à être cédés par leur acquéreur, la TVA qui a potentiellement grevé leur acquisition, du fait d'une option du vendeur, n' est pas déductible dès lors que cette acquisition ne peut, prima facie, être considérée comme un élément constitutif d'une opération taxée en aval ouvrant droit à déduction. S'agissant ici d'un immeuble non neuf, sa cession future ne sera en effet imposable que sur option du cédant. Telle est d'ailleurs la position de l'Administration qui précise que la taxe supportée au titre des frais attachés à la livraison d'un immeuble « est immédiatement déductible lorsque la vente ou l'apport concerne un terrain à bâtir ou un immeuble qui n'est pas achevé depuis plus de cinq ans dès lors que ces opérations sont imposables de plein droit. En revanche, lorsque la livraison concerne un terrain qui n'est pas à bâtir ou un immeuble achevé depuis plus de cinq ans, l'opération étant exonérée de plein droit, la taxe qui a grevé les frais en cause est réputée non déductible et, s’il y a lieu, ne peut être déduite qu'une fois effectivement exercée l'option dans les conditions prévues à l'article 201 quater de l'annexe II du CGI » 40.

Sur le second point, le juge considère qu'il n'existe pas de lien direct et immédiat entre l'achat de l'immeuble et l'activité de location qui n'est qu'intercalaire. Même si la décision n'est pas très loquace, la lecture des conclusions de Céline Guibé renseigne sur la démarche du juge. Le marchand de biens requérant ayant inscrit l'immeuble en stocks et souscrit un engagement de revendre sous cinq ans de l'article $1115 \mathrm{du}$ CGI, le Conseil d'État semble avoir considéré que l'immeuble, nonobstant le fait qu'il était loué, avait été dès son acquisition affecté à l'activité de revente du marchand de biens, la location n'étant qu'une modalité d'attente. Or réfutant, comme le lui suggérait son rapporteur public41, que cet immeuble puisse être considéré, pour les besoins de détermination de la TVA déductible, comme utilisé concurremment pour deux activités distinctes, une activité de vente et une activité de location, il a jugé que la location ne pouvait avoir pour effet de rompre le lien direct et immédiat créée entre l'acquisition de l'immeuble et l'activité de vente.

Au plan théorique, on peut néanmoins objecter que l'acquisition ou la construction d'un immeuble neuf ne garantit nullement qu'il conserve cette qualification jusqu'à sa vente, de sorte que la justification du traitement spécifique réservé à la TVA supportée lors de l'acquisition d'un immeuble non neuf parait bien 
fragile. Au plan économique, cette décision impose au professionnel le portage de la TVA jusqu'à la revente de l'immeuble, au mépris d'une conception qui aurait pu être plus respectueuse du principe de neutralité de la TVA 42. En tous les cas, elle encourage les marchands de biens à se défaire le plus rapide- ment possible de leurs stocks... Actant cette difficulté, le rapporteur public suggérait d'ailleurs au législateur d'ouvrir la possibilité pour les marchands de biens d'opter par anticipation pour la TVA sur la revente de leurs immeubles en stocks, à charge pour eux de reverser la TVA déduite si, in fine, leur vente n'était pas soumise à TVA.

\section{DROITS DE MUTATION À TITRE ONÉREUX}

Autre volet fiscal de la cession de biens immobiliers, les droits de mutation à titre onéreux ont connu un certain nombre d'évolutions dont il va maintenant être rendu compte.

\section{A. CESSIONS DE PARTICIPATIONS, APPRÉCIATION DE LA PRÉPONDÉRANCE IMMOBILIÈRE}

En application des dispositions de l'article $726, \mathrm{I}, 2^{\circ} \mathrm{du}$ CGI, les cessions de participations dans des personnes morales à prépondérance immobilière sont soumises aux droits de mutation à titre onéreux au taux de 5\% (au lieu de 0,10 ou 3\%). Selon ce même texte, la prépondérance immobilière caractérise la personne morale, dont les droits sociaux ne sont pas négociés sur un marché réglementé, dont l'actif est, ou a été au cours de l'année précédant la cession des participations en cause, principalement constitué d'immeubles ou de droits immobiliers situés en France ou de participations dans des personnes morales, elles-mêmes à prépondérance immobilière. La Cour de Cassation a, dans un arrêt du 2 décembre 2020, Min. c/Sté Établissement Bernard Escande et cie43, jugé que le texte du $2^{\circ}$ du I de l'article 726, qui ne vise que les immeubles et les droits immobiliers ne permet pas de prendre en compte les immeubles par destination de l'article 517 du Code civil pour apprécier la prépondérance d'une société dont les titres sont cédés. En l'espèce, était en cause la cession d'actions d'une société anonyme simplifiée exploitant une centrale électrique.

$B$.

ENGAGEMENT DE CONSTRUIRE ET DE REVENDRE

\section{a ) Justification de la réalisation des travaux}

Aux termes des dispositions du I du A de l'article 1594-0 G du CGI, l'acqué- reur assujetti d'un bien immobilier peut éviter le paiement des droits de mutation à titre onéreux de droit commun, en prenant l'engagement de réaliser dans les quatre ans des travaux conduisant à la production d'un immeuble neuf ou nécessaires pour terminer un immeuble inachevé. Normalement contracté dans l'acte d'acquisition, cet engagement peut, selon la doctrine administrative, également résulter d'un acte complémentaire enregistré auprès du service des impôts du lieu de situation de l'immeuble 44. C'est à propos de cette possibi- lité, fort utile lorsque l'acquéreur s'aperçoit postérieurement à son achat que l'ampleur des travaux à réaliser conduira à la production d'un immeuble neuf au sens du $2^{\circ}$ du 2 de $1^{\prime}$ article 257 du CGI, que la Cour de cassation a rendu une décision Sté Destrebis 45 le 29 janvier 2020. Bien qu'elle ne comporte pas de solution nouvelle, cette décision soulève néanmoins d'intéressantes questions. En premier lieu, elle valide, sans référence au BOFIP, la pratique des engagements différés alors même que l'article 1594-0 G du CGI précise expressément que l'engagement doit être contenu dans l'acte d'acquisition. Dans un autre domaine, celui des engagements de conservation donnant lieu à exonération partielle des droits de mutation à titre gratuit, la Cour avait pourtant jugé, par une interprétation littérale de l'article 787 B, c) du CGI, que l'absence de mention de l'engagement dans la déclaration de succession privait définitivement les héritiers de la possibilité de bénéficier de la réduction des droits d'enregistrement46. L'absence de référence au BOFIP peut s'expliquer par le fait que la possibilité de prendre un engagement de manière différée n'était pas contestée en l'espèce. Pour autant, si la Cour devait confirmer qu'une telle possibilité trouve son fondement 
dans l'article 1594-0 G du CGI, se poserait la question de son extension à l'engagement de revendre prévu à l'article 1115 du CGI, alors même que les commentaires administratifs ne la prévoient pas 47.

Les faits posent une seconde question. En l'hypothèse, à la date de l'acte comportant engagement de construire, les travaux auxquels s'étaient engagée la société acquéreuse avaient déjà en partie été réalisés, non par cette dernière mais par sa locataire. La cour d'appel 48 avait considéré que la réalisation des travaux de rénovation par ce tiers ne permettait pas de justifier de l'exécution des travaux visés par l'engagement souscrit par la société requérante et justifiait le refus de restitution des droit acquittés. La Cour de cassation ne s'est pas saisie expressément de la branche du moyen contestant ce raisonnement, mais a simplement rappelé que l'exonération est acquise dès lors que l'acquéreur assujetti a pris un engagement de construire dans les quatre ans de l'acte d'acquisition et ne peut être remise en cause « qu'à défaut de justification de leur réalisation à l'expiration de ce délai ». Ce faisant, il ne nous semble pas qu'elle ait, comme on a pu l'écrire49, validé la possibilité de justifier du respect d'un engagement de construire par la preuve de la réalisation des travaux par un tiers, en l'absence de reprise de l'engagement de l'acquéreur. Or, cette question est délicate car non seulement l'Administration procède à des rectifications sur ce motif comme en atteste l'affaire en question, mais à ce jour, elle a refusé de se prononcer expressément sur la question. Une réponse ministérielle «Sallé » 50 avait admis que le bénéfice de l'exonération prévue à l'ancien article 1371 du CGI n'était pas remis en cause dès lors que l'immeuble était édifié et achevé dans le délai de quatre ans à compter de son acquisition quand bien même lesdits travaux n'auraient pas été le fait de l'acquéreur. Cette réponse n'a pas été reprise au BOFIP, et les questions parlementaires ultérieures n'ont à ce jour pas obtenu de réponse 51.

On rappellera par ailleurs, que par une décision du 18 novembre 2019, n 18/09394, SAS Daumalis, la Cour d'Appel de Paris52 a confirmé que si l'acte d'engagement différé peut être déposé dans les quatre ans de l'acte d'acquisition, le reversement des droits correspondant doit être demandé avant le 31 décembre de la seconde année qui suit celle au cours de laquelle l'acte complémentaire comportant engagement de construire a été déposé. Elle a en effet jugé que le point de départ du délai de 2 ans de l'article R* 196-1 du LPF dans lequel doit être formulée la réclamation en ce sens se situe non pas à la date du versement des droits dont la restitution est demandée, comme le prétendait l'Administration, mais à la date de dépôt de l'acte complémentaire comportant engagement de construire. Outre que cette décision semble conforme à la doctrine administrative qui considère que constitue un événement de nature à rouvrir le délai de réclamation au sens de l'article $\mathrm{R}^{*} 196-1$, première partie, c) du LPF tout fait « ayant pour effet de modifier rétroactivement l'assiette ou le calcul de cette imposition » 53, il s'agit en outre de la seule solution qui permette d'assurer l'effectivité de l'acte complémentaire déposé dans le délai de quatre ans de l'article 1594-0 G du CGI.

\section{b ) COVID, suspension du délai pour construire ou revendre}

L'article 8 de l'ordonnance $\mathrm{n}^{\circ} 2020-306$ du 25 mars 2020 relative à la pro- rogation des délais échus pendant la période d'urgence prévoit que « lorsqu'ils n'ont pas expiré avant le 12 mars, les délais imposés par l'Administration, conformément à la loi et au règlement, à toute personne pour réaliser des contrôles et des travaux ou pour se conformer à des prescriptions de toute nature sont, à cette date suspendus jusqu'à la fin de la période mentionnée au I de l'article ler, sauf s'ils résultent d'une décision de justice. Le point de départ des délais de même nature qui auraient dû commencer à courir pendant la période mentionnée au I de l'article ler est reporté jusqu'à l'achèvement de celle-ci ».

Sur le fondement de ce texte, l'Administration fiscale 54 a admis que les délais imposés pour le respect des engagements de revendre de l'article 1115 du CGI, ou de construire de l'article 1594-0 G du même code, qui n'étaient pas expirés avant le 12 mars 2020 ont été suspendus pendant toute la période juridiquement neutralisée, c'est-à-dire jusqu'au 23 juin 2020 inclus. Il en résulte que les assujettis pour lesquels le délai pour construire venait normalement à expiration au 12 mars 2020 ou à une date ultérieure ont disposé ou 
disposent d'un délai supplémentaire de 104 jours pour satisfaire à leurs obligations. Pour ceux ayant souscrit un engagement de revendre ou de construire pendant la période protégée, c'est-à-dire entre le 12 mars et le 23 juin 2020, le délai de cinq ou de quatre ans selon cas, n'a commencé à courir que le 24 juin 2020 et non à la date de l'acte d'acquisition.

\section{c ) Contrôle du respect de l'engagement de construire}

En principe, et aux termes même de l'article 1594-0 G du CGI, l'exonération est subordonnée à la condition que l'assujetti justifie à l'expiration du délai de quatre ans dans les conditions du $2^{\circ}$ du I de l'article 266 bis de l'annexe III du CGI de l'exécution des travaux auxquels il s'est engagé par le dépôt en mairie d'une déclaration d'achèvement des travaux55. Si cette déclaration n'est pas déposée et si l'assujetti n'a pas spontanément procédé à la liquidation et au paiement des droits, l'Administration peut exiger leur paiement sous réserve d'agir dans son délai de reprise. Ce délai commence à courir à compter du jour où l'Administration peut agir valablement à l'encontre du redevable, à savoir non pas à la date de l'acte initial mais à celle à laquelle a expiré le délai dont disposait l'acquéreur pour construire56. Pour les assujettis qui auront bénéficié de la suspension du délai qui leur était imparti en application de l'ordonnance du 25 mars 2020 susvisée, le délai de reprise de l'administration commencera donc à courir à la date d'expiration du délai tel que prorogé.

Certains contribuables ont par ailleurs pu soutenir qu'en l'absence de dépôt de la déclaration d'achèvement des travaux, l'action de l'Administration se trouverait enfermée dans le délai triennal de l'article L.180 du LPF. Ils soutenaient que le non dépôt de cette déclaration suffisant à justifier des rectifications, l'administration n'a pas à procéder aux « recherches ultérieures », excluant, selon ce même texte, l'application de la prescription triennale pour notifier des rectifications 57. La Cour de cassation n'a pas suivi cette analyse et a jugé, dans un arrêt du 7 juillet 2020, $\mathrm{n}^{\circ}$ 18-10. 525 D, SCI Les Aravis, que lorsqu'aucune démarche révélatrice de l'exigibilité de droits supplémentaires, dépôt de la déclaration d'achèvement ou demande de prorogation du délai initial, n'a été effectuée auprès de l'Administration pour l'aviser du non achèvement des travaux, la prescription sexennale de L.186 du LPF s'applique. Cette position est particulièrement rigoureuse. Elle s'appuie sur le texte de l'article L.180 du LPF qui précise qu'en matière de droits d'enregistrement la prescription triennale est opposable à l'Administration si l'exigibilité des droits a été suffisamment révélée par un document enregistré ou présenté à la formalité58. Cette décision confirme la sévérité de la jurisprudence relative au suivi des engagements de construire et invite en conséquence les assujettis à la plus grande rigueur en la matière.

\section{Notes de bas de page}

1. BOI-TVA-CHAMP-50-10, 4 avril 2014, $\$ 120$.

2. CE, 8e et 3e ch. réunies, 9 sept 2020, n439143, Sté (SCI) EMO : Construction - Urbanisme 2020, comm. 117 ; RJF 12/20, c. 989.

3. BOI-TVA-SECT-80-50-10, 4 avr. 2014, §1.

4. Dans une décision du 22 octobre 2020, $\mathrm{n}^{\circ}$ 18NT03655, EURL Bazille, la CAA de Nantes a jugé que l'engagement pris par le cédant dans l'acte d'acquisition de poursuivre la location en TVA ne vaut pas option pour la TVA, de sorte que la cession n'ayant pas été réalisée entre deux assujettis, la dispense doit être remise en cause. En l'espèce, il semble que la requérante n'avait pas entendu se prévaloir de la possibilité de reprendre l'option de son cédant (BOI-TVA-CHAMP-50-10, 4 avr. 2014, §240).

5. CA Montpellier, 22 mai 2020, $n^{\circ} 16 / 01365$, BPAT 4/20, 129 ; note E. Cruvelier, Loyers et Copropriété, 2020, $\mathrm{n}^{\circ}$ 7-8, alerte 37. En l'hypothèse, en revanche, la responsabilité du notaire ne pouvait 
être engagée, celui-ci n'ayant pas manqué à son devoir d'information. Voir pour une solution inverse : CA Paris, 22 fév. 2019, n 17/10059.

6. Sur les questions relatives à l'application dans le temps de la jurisprudence SCI EMO, voir

G. Berger-Picq, «Locations de locaux nus à usage professionnel : une option pour la TVA sur mesure », FR 40/20, 4 ; F. Alparslan, A. Herbelot, S. Laurent-Sorel, «Option pour la TVA sur la location nue de locaux professionnels : le bailleur est délivré du tout ou rien » : Dr. fisc. 2020, n 41, comm. 400.

7. CE, 9 juin 2020, $\mathrm{n}^{\circ}$ 432596, RJF 8-9/2020, c. 686.

8. CJUE, 2e ch., 15 sept. 2011, aff. C-180/10 Slaby et aff. C-181/10 Kué, note W. Stemmer : Dr. fisc. 2011, $\mathrm{n}^{\circ} 48$, comm. 608

9. BOI-TVA-IMM-10-10-10-10, 12 sept. 2012, §20.

10. Particulièrement point 46 de l'arrêt. Illogisme pointé par W. Stemmer qui notait dans ses commentaires sous l'arrêt Slaby (loc. cit.) qu' « un État membre qui n’a pas usé de la faculté de taxer « un assujetti occasionnel » devrait avoir plus de difficulté à taxer une opération ponctuelle de lotissement ».

11. Dans une hypothèse similaire à celle transmise au conseil d'État, la Cour d'appel de Bordeaux a considéré que les conditions de l'assujettissement n'étaient pas présentes, à défaut pour le vendeur d'avoir mis en œuvre les moyens commerciaux utilisés par les professionnels de la vente immobilière : CAA, Bordeaux 7e ch., 20 mai 2020, 18 BX01002.

12. BOI-TVA-IMM-10-10-10-10, 12 sept. 2012, § 90.

13. Note W. Stemmer et M. Corabouef sous CAA Lyon, 2e ch., 27r 2014, n 13LY00739,

M. et Mme Caron : Dr. fisc. 2014, $\mathrm{n}^{\circ}$ 37, comm. 521.

14. BOI-RES-000064, 28 oct. 2020.

15. BOI-TVA-IMM-10-10-10-20, 29 sept. 2014, $§ 120$ et $\$ 300$

16. Voir commentaire de N. Gonzalez Gharbi : Construction - Urbanisme 2020, $\mathrm{n}^{\circ} 12$, comm. 147.

17. BOI-TVA-IMM-10-10-20, 2 mars 2016, § 270.

18. Pour ce dispositif voir BOI-TVA-IMM-30, 28 oct. 2020.

19. Sur ces conditions voir BOI-TVA-IMM-30, 19 fév. 2020.

20. Pour un commentaire de ces conditions : BOI-TVA-IMM-10-20-10, 13 mai 2020.

21. Par exemple : RM Savary, n $^{\circ} 94538$, JO AN 20 oct. 2016 ; RM Bussereau, $n^{\circ} 96679$, JO AN 20 oct. 2016.

22. TA Grenoble, 14 nov. 2016 ; TA Montpellier, 4 déc. 2017, n 1602770 ; CAA Lyon, 20 déc. 2018, n²17Y03359.

23. RM Vogel, n $^{\circ}$ 04171, JO Sénat 17 mai 2018, p. 2361.

24. RM Falorni, ${ }^{\circ} 1835$, JO AN 24 sept. 2019, p. 8300.

25. Directive 2006/112/CE, art. 392 : les États membres peuvent prévoir que, pour les livraisons de bâtiments et de terrains à bâtir achetés en vue de la revente par un assujetti qui n'a pas eu droit à déduction à l'occasion de l'acquisition, la base d'imposition est constituée par la différence entre le prix de vente et le prix d'achat.

26. CE, 27 mars 2020, n 428234, SARL Promialp, JCP N. 2020, $\mathrm{n}^{\circ}$ 24, 1130.

27. CE, 8e et 3e ch, 16 juill. 2020, n 435464, concl. K. Civaldin : Dr. fisc. 2020, $\mathrm{n}^{\circ} 45$, comm. 429.

28. RM Bazin, $n^{\circ} 30767$, JO AN 29 sept. 2020.

29. CE, 30 novembre 2020, $\mathrm{n}^{\circ} 440137$, SARL Les Avaris.

30. BOI-TVA-IMM-10-10-10-40, 7 janv. 2013, $§ 10$ : « en principe et pour l'application de la TVA, l'acte doit refléter clairement la qualification respective de chaque élément, en sorte que le régime approprié soit distinctement et régulièrement appliqué ». L'administration admet néanmoins que puisse, en TVA, être vendu en tant qu'immeuble bâti « la partie du terrain formant dépendances indispensables et immédiates des bâtiments (cours, passages, jardins de moins de cinq ares, etc.) ».

31. BOI-TVA-IMM-10-10-10-20, 29 sept. 2014, § 110.

32. Par exemple, RM Bussereau, $\mathrm{n}^{\circ} 96679$ JO AN 31 août 2016 : « lorsque la division parcellaire est antérieure à l'acte d'acquisition initial, qu'un document d'arpentage a été établi pour les besoins de la cession permettant d'identifier les différentes parcelles dans l'acte ou qu'un permis d'aménager faisant apparaître de manière précise les divisions envisagées a été obtenu préalablement à la cession, la taxation 
sur la marge s'applique dès lors qu'aucun changement physique ou de qualification juridique des parcelles cédées n'est intervenu avant la revente $\gg$.

33. BOI-TVA-IMM-10-20-10, 13 mai 2020, §30.

34. CE, 3e et 8e ch, 25 juin 2020, n 416727, Sté Icade Promotion logement : Dr. fisc. 2020, n³0-35, comm. 325 ; N. Gonzalez Gharbi, «TVA sur marge et revente de terrains à bâtir : le Conseil d'État préfère la question préjudicielle à la question prioritaire de constitutionnalité », Construction-urbanisme 2020, $\mathrm{n}^{\circ} 9$, comm. 100.

35. BOI-TVA-IMM-10-20-10, 13 mai 2020, $\$ 300$.

36. CAA de Bordeaux, 5e ch., 9 juil. 2020, 18BX02878, Société Soc Commerciale Industrielle Immobilière.

37. CAA Lyon, 25 juin 2019, $\mathrm{n}^{\circ}$ 18LY00671, Construction-Urbanisme 2019, $\mathrm{n}^{\circ}$ 9, comm. 115.

38. CE, 9e et 10e ch., 27 nov. 2020, n 426091, Sté Lips et Financière Lord Byron, Dr. fisc. $2020, \mathrm{n}^{\circ} 49$, act. 429.

39. CGI art. 271, I : CJUE Cibo Participations, 27 sept. 2000, aff. C 16/00, point 31; CJUE AB-SKF, 29 oct. 2009, aff. C-29/08, RJF 1/10, n $^{\circ} 90$.

40. BOI-TVA-IMM-10-30, 2 mars 2016, § 80.

41. Conclusions C. Guibé, 27 nov. 2020, $n^{\circ}$ 426091, Arianeweb.

42. Marc Wolf, « La neutralité, ce n'est pas « plus tard » : retour sur la déduction de la TVA d'achat des immeubles anciens », Étude, BF 1/20.

43. Cass. com., 2 déc. 2020, $\mathrm{n}^{\circ} 18-25.559$, min. c/ Sté Etablissement Bernard Escande et cie :

Dr. fisc. 2020, $\mathrm{n}^{\circ} 50$, act. 453.

44. BOI-ENR-DMTOI-10-40, 1er juin 2016, § 270.

45. Cass. Com, 29 janv. 2020, $\mathrm{n}^{\circ}$ 17-26.018, Sté Destrebis ; Construction-Urbanisme 2020, $\mathrm{n}^{\circ}$ 6, comm. 73 avec note Neyla Gonzalez-Gharbi, «Engagement de construire : un temps pour le souscrire et un autre pour justifier de son respect ».

46. Cass. civ., 16 avr. 2013, 12-17.432.

47. BOI-ENR-DMTOI-10-50, 29 avr. 2014.

48. CA Saint Denis-Denis de la Réunion, 30 juin 2017, Ch. civ., $n^{\circ}$ 15/01645.

49. A. Moraine, "Les conditions de l'engagement de construire portant exonération des droits d'enregistrement précisées par la Cour de Cassation », JCP N. 2020, n 13, 1078.

50. RM. Sallé, n 4451, JOAN 14 juin 1969.

51. Q. Fasquelle, n 100632 : JOAN 15 nov. 2016 ; Q. Morel-à-L’Huissier, n 24746, JOAN 26 nov. 2019 ; Q. Dallier, n07912, JO Sénat 29 nov. 2018 p. 5960.

52. Intervenant en appel de TGI Paris, 27 mars 2018, $\mathrm{n}^{\circ}$ 15/16226.

53. BOI-CTX-PREA-10-30, 12 sept. 2012, §60.

54. BOI-DJC-COVID19-10, 24 juin 2020, $\$ 110$.

55. BOI-ENR-DMTOI-10-40, 1 juin 2016, §410.

56. Cass. com., 31 oct. 2006, $\mathrm{n}^{\circ}$ 05-10396 ; Cass. Com, 22 nov. 2016, $\mathrm{n}^{\circ}$ 15-14.797.

57. D'autant que certaines Cour d'appel considèrent que le dépôt de la déclaration d'achèvement des travaux est une condition de fond de l'exonération, indépendamment de la réalisation effective des travaux : V. CA Reims, 23 avril 2019, n 18/011655.

58. Cette solution confirme celle retenue dans Cass. Com., 3 avril 2012, $\mathrm{n}^{\circ} 11-13.869$, F-D, Sté Les Vergniouds, Dr. fisc. 2012, $\mathrm{n}^{\circ}$ 26, comm. 357. La motivation était cependant différente, la Cour ayant dans cette précédente affaire motivé l'application du délai de droit commun par le fait que «l'administration ne peut avoir connaissance de l'évènement futur et incertain que constitue la réalisation de constructions que par des recherches ultérieures, en l'occurrence l'examen de documents cadastraux ». 